\title{
Acknowledgment to Reviewers of Climate in 2020
}

\section{Climate Editorial Office}

Citation: Climate Editorial Office.

Acknowledgment to Reviewers of Climate in 2020. Climate 2021, 9, 24. https://doi.org/10.3390/cli9020024

\section{Published: 24 January 2021}

Publisher's Note: MDPI stays neutral with regard to jurisdictional claims in published maps and institutional affiliations.

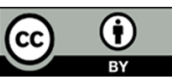

Copyright: (c) 2021 by the author. Licensee MDPI, Basel, Switzerland. This article is an open access article distributed under the terms and conditions of the Creative Commons Attribution (CC BY) license (http://creativecommons.org/licenses /by/4.0/).

MDPI AG, St. Alban-Anlage 66, 4052 Basel, Switzerland

Peer review is the driving force of journal development, and reviewers are gatekeepers who ensure that Climate maintains its standards for the high quality of its published papers. Thanks to the cooperation of our reviewers, in 2020, the median time to first decision was 16 days and the median time to publication was 38 days. The editors would like to express their sincere gratitude to the following reviewers for their precious time and dedication, regardless of whether the papers were finally published:

Abbas, Jaffar

Abbas, Sawaid

Abou Rafee, Sameh A.

Acharya, Bharat

Adhikari, Shankar

Adnan, Sarfaraz

Afionis, Stavros

Agathokleous, Evgenios

Aguilos, Maricar

Ahamad, Mazbahul

Ahmed, Nesar

Akbari, Hashem

Alauddin, Mohammad

Alghamdi, Ali

Allen, Melissa Ree

Al-Musaed, Amjad

Amantov, Aleksey

Anderbrant, Olle

Andrade, Felipe M. De

Andrei, Simona

Añel, Juan Antonio

Antwi Agyei, Philip

Aranda, Xavier

Argilaga, Albert

Armani, Gustavo

Artale, Vincenzo

Aryal, Yog

Aslam, Muhammad

Asumadu-Sarkodie, Samuel

Auger, Jeffrey D.

Avila, Alvaro Javier

Ayzel, Georgy

Babich, Francesco

Babovic, Vladan

Baker, Jess

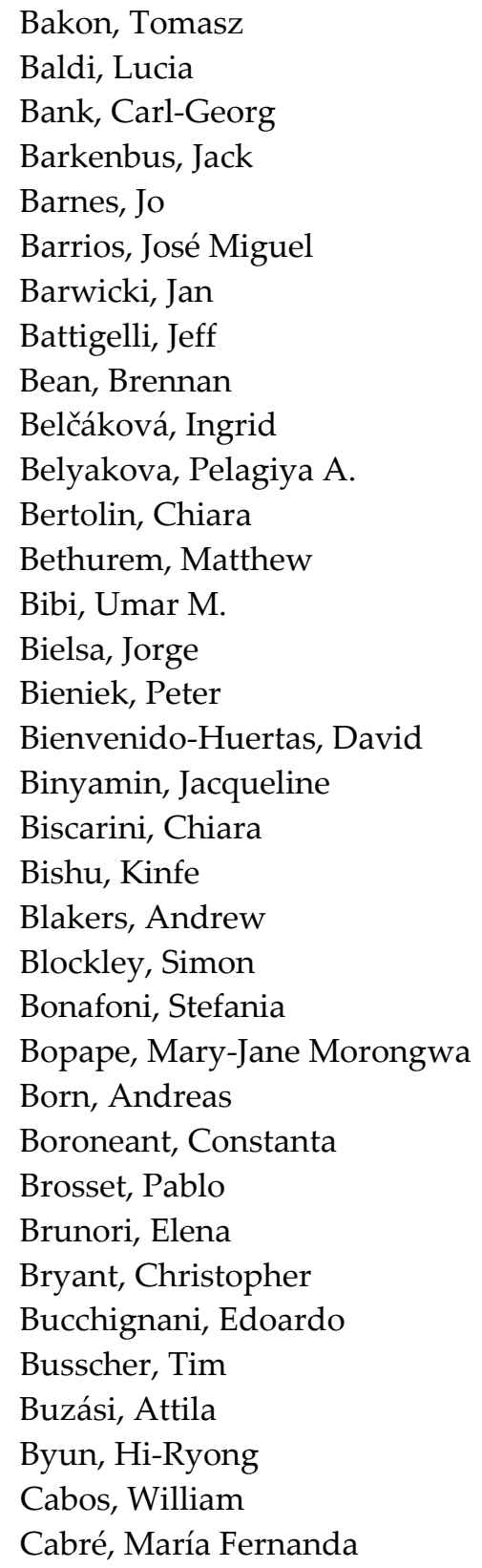

Cabré, María Fernanda 
Calanca, Pierluigi

Caloiero, Tommaso

Calvo-Alvarado, Julio César

Campbell, Carol

Caridade, Pedro Jorge Dos Santos Branco

Carlsen, Hanne Krage

Cartalis, Constantinos

Cary, Michael

Caton, Kellee

Cazacu, Constantin

Cebulska, Marta

Chaplot, Vincent

Charalampopoulos, Ioannis

Chattopadhyay, Somsubhra

Chatzidimitriou, Angeliki

Chau, Kwok-Wing

Chemura, Abel

Chojnacka-Ożga, Longina

Chowdhury, AFM Kamal

Chun, Jong Ahn

Ciampittiello, Marzia

Clark, Daniel G.

CLAVERIE, Remy

Clay, Patricia M.

Colaiuda, Valentina

Comarazamy, Daniel

Constanta Zoie, Radulescu

Constantin, Daniel-Eduard

Cordeiro, Marcos

Corney, Stuart

Cui, Yuyan

Curtis, Scott

Daly, Cathy

Danza, Ludovico

Davis, W. Jackson
Doutreloup, Sébastien

Drąg, Paweł

Dulebenets, Maxim A.

Ediriweera, Sisira

Edwards, Keith

Eensaar, Agu

El Kenawy, Ahmed

Emanuelsson, B. Daniel

Emmerling, Johannes

Engstrom, Johanna

Escobar, Yesid Carvajal

Estévez, Javier Gualda

Facão, Jorge

Falasca, Serena

Fang, Keyan

Fang, Yuanhao

Farnham, Craig

Favretti, Marco

Feher, Alexander

Fei, Huo

Fekete, Alexander

Felzer, Benjamin S.

Fernandez, Sergio

Fernández-Manjarrés, Juan F.

Fiorito, Francesco

Fita, Lluís

Fletcher, Amber

Flocas, Helena

Fornaro, Adalgiza

Fossum Evertsen, Kathinka

Fosu, Boniface

Foti, Giandomenico

Fouladi, Kamran

Franklin, Charmaine

Freni, Gabriele

De Costa Trindade Amorim, Margarete CristianeFu, Weiwei

De Figueiredo Neves, Gustavo Zen

De La Casa, Antonio

De Morais, Marcos Vinicius Bueno

De Santi, Francesca

Deelstra, Johannes

Den, Walter

Deng, Chenda

Deng, Jifeng

Dhakal, Chandra

Fullerton, Aimee $\mathrm{H}$.

Fusco, Giulio

Gadedjisso-Tossou, Agossou

Galán De Mera, Antonio

Galdies, Charles

Galiatsatou, Panagiota

Gallus, William A.

Galvani, Emerson

Galve, Joan Miquel

Garza-Rodríguez, Iliana De La

Gavrilov, Milivoj B.

Gentilucci, Matteo

Georgiadis, Teodoro

Ghafouri-Azar, Mona

Gharehbaghi, Koorosh

Dos Santos Silva, Fabrício Daniel 
Gialampoukidis, Ilias

Gilja, Gordon

Gil-Pelegrín, Eustaquio

Glazebrook, P. (Tricia)

Gobo, João Paulo Assis

Golding, Brian

Goodarzi, Mohsen

Gourbesville, Philippe

Grant, Bruce

Gribanov, K. G.

Grigorieva, Elena

Grunig, Gabriele

$\mathrm{Gu}$, Jiangxin

Gulbeyaz, Onder

Gusarov, Artyom (Artëm)

Gutiérrez López, Alfonso

Gutiérrez, Edgar

Güttler, Ivan

Hadjinicolaou, Panos

Halbac-Cotoara-Zamfir, Rares

Hammock, Bruce G.

Hamududu, Byman Hikanyona

Han, Daehyeon

Hansen, Sissel

Hantush, Mohamed M.

Hanzl, Małgorzata

Harrington, John A.

Hatfield, Samantha Chisholm

He, Baojie

Hellmund, Tobias

Hernández Ayala, José Javier

Hernández-Pérez, Iván

Hewelke, Edyta

Hidy, George

Hirano, Yujiro

Hocke, Klemens

Hoffmann, Sharon

Hoppe, Thomas

Horodyskyj, Ulyana Nadia

Hssaisoune, Mohammed

$\mathrm{Hu}$, Caihong

$\mathrm{Hu}$, Tangao

Huang, Bo

Huang, Jr-chuan

Humpe, Andreas

Iglesias, Isabel

Ikeuchi, Hiroaki

Im, Sangjun

Ingwersen, Joachim

Ippoliti, Carla

Isaac, Shabtai
Isaksson, Charlotta

Islam, Kazi Kamrul

Iyer-Raniga, Usha

Jacobs, Brent

Jang, Min-Won

Jato-Espino, Daniel

Jessup, Stephen

Jimenez Rodriguez, Cesar Dionisio

Jin, Liya

Johansson, Eva

Johnson, Zachary F.

Joshi, Niraj Prakash

Kalantar, Bahareh

Kambezidis, Harry D.

Kamiński, Jacek Wojciech

Karatassiou, Maria

Kariuki, Rebecca

Karki, Tej

Kausrud, Kyrre

Kavoosi, Masoud

Kayad, Ahmed

Kazandjiev, Valentin

Kelletat, Dieter

Khader, Abdelhaleem

Khan, Asim

Kibet, Leonard

Kiełtyka-Dadasiewicz, Anna

Kikuchi, Maki

Kim, Jin-Soo

Kirby, Mac

Kishcha, Pavel

Kluczkovski, Alana

Kobiv, Yuriy

Koloutsou-Vakakis, Sotiria

Korennoy, F. I.

Kovács, Ferenc

Kovalčík, Miroslav

Kozubek, Michal

Križan, Peter

Krstanović, Vinko

Krzyszczak, Jaromir

Kuhn, Michael

Kukal, Meetpal

Kumar, Nallapaneni Manoj

Kumar, Praveen

Kumar, Vinay

Kundzewicz, Zbigniew W.

Kuriqi, Alban

Kwak, Kyung-Hwan

Lacko, Roman

Lahmers, Timothy 
Lai, Derrick Y.F.

Landelius, Tomas

Lanza, Kevin

Laskin, David

Lazoglou, Georgia

Lee, Dong Eun

Lee, Yang-Won

Lepczyk, Christopher

Lesschen, Jan Peter

Letchov, George

Levi, Yoav

Li, Haiqin

Li, Huidong

Li, Laurent Z.X.

Li, Ming Hsu

Li, Sanai

Li, Xianxiang

Li, Xiujun

Li, Ying

Liang, Boyi

Lin, Zihan

Liobikienè, Genovaite

Lira-Loarca, Andrea

Lirer, Fabrizio

Littlefield, Jeffrey

Liu, Changjun

Liu, Duanyang

Liu, Qiang

Liu, Tie

Liu, Wen-Cheng

Liu, Yaqun

Liu, Yonggang

Lo Faro, Alessandro

Loehle, Craig

Louis, Valérie R

Loukas, Athanasios

Lovino, Miguel

Lowman, Lauren

Lucille, Alonso

Lujala, Paivi

Lund, Steve

Lupo, Anthony R.

Lykhovyd, Pavlo

Ma, Weiqiang

Ma, Yingzhao

Machar, Ivo

Mackay, J.D.

Mahar, Waqas Ahmed

Mahdu, Omchand

Maheng, Dikman

Maiolo, Mario
Mallakpour, Iman

Mallory, Ellen B.

Maloney, Eric D.

Malyan, Sandeep

Manousos, Manousakas

Marco, Delle Rose

Maresi, Giorgio

Marianna, Nardino

Martinho, Vítor João Pereira Domingues

Mascher, Sharon

Masia, Sara

Mata-Lima, Herlander

Mataveli, Guilherme Augusto Verola

Mathbout, Shifa

Matic, Frano

Matiu, Michael

Matsui, Kenichi

Matthews, Damon

Matul, Alexander

Mavromatis, Theodoros

Mazza, Alessandro

Mazzarella, Adriano

McGrath, Gavan

Mehan, Sushant

Meldrum, Gennifer

Meléndez-Ackerman, Elvia

Mendez-Tejeda, Rafael

Mercer, Andrew

Mezei, Pavel

Michalski, Grzegorz

Michelsen, Teresa

Mihu-Pintilie, Alin

Milosevic, Dragan

Minoli, Sara

Mischler, John

Moazamigoodarzi, Hosein

Moeletsi, Mokhele E.

Mohammadi, Babak

Moncada, Stefano

Mondino, EB

Moodley, Desika

Moon, Byung-Kwon

Morille, Benjamin

Morrison, Alastair M.

Mosavi, Amir

$\mathrm{Mu}$, Jianhong

Mughal, Muhammad Omer

Mukherjee, Srijib K

Mukwada, Geoffrey

Munir, Tariq M.

Muscio, Alberto 
Musco, Francesco

Myslenkov, Stanislav

Nafiseh, Haghtalab

Nagy, Gustavo

Najibi, Nasser

Narapusetty, Balachandrudu

Nardino, Marianna

Nayak, Sridhara

Ndoro, Jorine

Neupane, Dhurba

Nguyen, Kim-Anh

Nguyen, Thao Phuong

Nooni, Isaac

Nunes, Leonel Jorge Ribeiro

Nursey-Bray, Melissa

Okoro, Stanley U.

Palme, Massimo

Panda, Sudhanshu Sekhar

Paneque, Manuel

Papaefthimiou, Spiros

Paranunzio, Roberta

Park, Jongmin

Park, Moojong

Park, Sunjoo

Parsaee, Mojtaba

Partanen, Antti-Ilari

Pasken, Robert

Patlakas, Platon

Patro, Epari Ritesh

Peña, Carlos

Perdana, Aji Putra

Pereira, Mário Gonzalez

Pereira, Susana

Perry, Jim

Petersen, Lillian Kay

Petrucci, Enrica

Peugeot, Christophe

Piccolo, Katherine

Pielech, Remigiusz

Pielke, Roger

Pinault, Jean-Louis

Pinto, Manuel

Piotrowski, Piotr

Pivić, Radmila

Popa, Bogdan

Pope, Katherine S

Poškus, Mykolas Simas

Př́ivara, Andrej

Rahman, Habib

Rajib, Adnan

Rak, Janusz
Ranagalage, Manjula

Rao, Jian

Rasilla, Domingo

Ratnam, Venkata

Reese, Gerhard

Reichenau, Tim G.

Reinmuth, Evelyn

Reinsberg, Bernhard

Renard, Florent

Riccò, Matteo

Rico, Arantza

Rigo, Tomeu

Rodrigo-Comino, Jesus

Rodrigues, Mónica

Rodriguez-Serrano, Enrique

Rohse, Melanie

Roman, Celian

Rotunno Filho, Otto Corrêa

Roundy, Paul

Ruiz-Alvarez, Osias

Ryoo, Ju-Mee

S. Biudes, Marcelo

Sa'adi, Zulfaqar

Sacchi, Barbara

Saha, Gopal Chandra

Sahu, Netrananda

Saifullah, Muhammad

Saksena, Siddharth

Salamon, Peter

Sales, Fernando De

Salvati, Paola

Samanta, Dhrubajyoti

Sanchez De La Flor, Francisco José

Sangelantoni, Lorenzo

Santarelli, Maria Laura

Santiago, José María

Sarker, Md Nazirul Islam

Scafetta, Nicola

Scaife, Adam A.

Scalisi, Alessio

Schlesinger, William

Schmutz, Vaughn

Schofield, Gail

Schüßler, Dominik

Scorzini, Anna Rita

Seebens, Hanno

Seip, Knut Lehre

Sekertekin, Aliihsan

Semedo, Alvaro

Sempi, Carlo

Senent-Aparicio, Javier 
Severini, Simone

Shafiei Shiva, Javad

Shahid, Shamsuddin

Shang, Songhao

Shannon, Kelly

Sharifi, Ehsan

Sharma, Vivek

Shaw, Rajib

Shen, Jingchun

Shiferaw, Andualem

Shin, Chul-Su

Shojafar, Mohammad

Shouzhi, Chang

Shrestha, Maheswor

Shrestha, Narayan

Shuai, Wang

Siderius, Christian

Sidorchuk, Aleksey

Siems, Steven

Skelhorn, Cynthia

Skender, Ana

Skjelvåg, Arne Oddvar

Soldatenko, Sergei

Soldatenko, Sergei A.

Song, Cholho

Song, Qingjun

Sousa, Daniel

Spiliotopoulos, M.

Spiliotopoulos, Marios

Stankovic, Milan

Starzec, Mariusz

Stathis, Dimitrios

Stechmann, Sam

Steinhoff, Daniel

Steinke, Valdir A.

Sthel, Marcelo Silva

Storchi, Paolo

Streimikiene, Dalia

Su, Yuan-Fong

Sullivan, Arnold

Sun, Xia

Swapan, Mohammad

Szabó-Takács, Beáta

Szturc, Jan

Tanner-McAllister, Sherri

Tantet, Alexis

Tegegne, Getachew

Tehrani, Mahsa Jahandideh

Teixeira, Danielle Cardozo Frasca

Teslic, Nemanja

Thayer, Anastasia W.
Thual, Sulian

Tiefenbacher, John P.

Tiepolo, Maurizio

Tîncu, Roxana

Toderi, Marco

Toth, Travis D.

Tsuge, Takahiro

Tsuruta, Aki

Tu, Chuan-Chi

Tuladhar, Dinesh

Tylkowski, Jacek

Tyralis, Hristos

Valdez Juárez, Luis Enrique

Valipour, Mohammad

Van Den Hurk, Bart

Varentsov, Mikhail

Varotsos, Costas

Vasseur, Liette

Vatolkina, Natalia

Vich Ramis, Maria-del-Mar

Vincenot, Christian

Viscione, Nunzio

Vitale, Marcello

Volenzo, Tom Elijah

Wang, Chenghao

Wang, Die

Wang, Tongli

Wang, Xander

Wang, Yupeng

Wang, Zheng Bing

Warner, Douglas

Wdowikowski, Marcin

Wei, Shanshan

Wesselbaum, Dennis

Wewerinke-Singh, Margaretha

Whanhee, Lee

Wheeler, Stephen

Williamson, Phillip

Wolski, Piotr

Woolliams, Emma

Work, Courtney

Wu, Chin-Lung

$\mathrm{Wu}, \mathrm{Shu}$

Wu, Wei

Wypych, Agnieszka

Xiao, Jieling

Ximenes, Fabiano

Xiong, Yongzhu

$\mathrm{Xu}$, Iris

$\mathrm{Xu}$, Yangyang

Yang, Chi 
Yang, $\mathrm{Hu}$

Yang, Meijian

Yang, Xiaodong

Yang, Yongzeng

Yannopoulos, Panayotis C.

Yap, Eng Hwa

Ye, Liming

Yoon, Sunkwon

Younus, Md

Yufei, Zou

Yurganov, Leonid

Zabret, Katarina

Zarzar, Chris

Zeng, Shihong
Zepeda, Eduardo

Zeroual, Ayoub

Zhang, Chen

Zhang, Huanxin

Zhang, Lei

Zhang, Wen

Zhang, Xingxing

Zhao, Tian

Zhao, Wei

Zhu, Laiyin

Ziaja, Wieslaw

Zittis, George

Zúñiga, Emmanuel 